\title{
Size-dependent free solution DNA electrophoresis in structured microfluidic systems
}

\author{
Thanh Tu Duong ${ }^{\mathrm{a}}$, Gyuman Kim ${ }^{\mathrm{b}, 1}$, Robert Ros ${ }^{\mathrm{a}}$, Martin Streek ${ }^{\mathrm{c}}$, \\ Friederike Schmid ${ }^{\mathrm{c}}$, Jürgen Brugger ${ }^{\mathrm{b}}$, Dario Anselmetti ${ }^{\mathrm{a}}$, Alexandra Ros ${ }^{\mathrm{a}, *}$ \\ ${ }^{a}$ Experimental Biophysics, Physics Department, Bielefeld University, Bielefeld, Germany \\ ${ }^{\mathrm{b}}$ Microsystems Laboratory, École Polytechnique Fédérale de Lausanne, Lausanne, Switzerland \\ ${ }^{\mathrm{c}}$ Condensed Matter Theory, Physics Department, Bielefeld University, Bielefeld, Germany
}

\begin{abstract}
A new concept based on microstructuring techniques is presented to achieve size-dependent electrophoretic migration of DNA in free solution. Topographically structured microfluidic channels with periodical cavities in the range of the radius of gyration of the tested DNA molecules $(<3 \mu \mathrm{m})$ are produced by moulding of polydimethylsiloxane on a master wafer fabricated by contact lithography of SU-8 photoresist. The electrophoretic migration of single DNA molecules stained with the intercalator YOYO was investigated by real-time fluorescence video microscopy. It could be demonstrated for large DNA molecules that $\lambda$-DNA (48 kbp) always migrated significantly faster than T2-DNA $(164 \mathrm{kbp})$ in the two tested microchannel layouts. Integration into a microdevice for DNA separation in free solution is now in progress.
\end{abstract}

Keywords: DNA; Electrophoresis; Microchip; Contact lithography; Single molecule; Fluorescence microscopy

\section{Introduction}

DNA electrophoresis represents a very important tool in molecular biology and genome analysis. With respect to the length independent mobility of DNA molecules in free solution, DNA electrophoresis is commonly carried out in gels. To enhance throughput and to shorten analysis time electrophoretic separation of DNA in capillaries or on microchips has been developed. However, the transfer of DNA separation to the microchip format has only been achieved for analytical applications in distinct DNA size ranges, as for example DNA sequencing [1-5]. This is mainly due to technical

\footnotetext{
*Corresponding author.

E-mail address: alexandra.ros@physik.uni-bielefeld.de (A. Ros).

${ }^{1}$ Present address: School of Mechanical Engineering, Kyungpook National University, 702-701 Daegu, Korea.
} 
problems related to the transfer and integration of conventional technology, e.g., the incorporation of cross-linked or high viscosity gels into capillaries and microchips [6].

Fluorescence microscopy with ultra-sensitive cameras allows the investigation of single (usually with fluorescent intercalators) labelled DNA molecules. This has been used by several groups to enlighten DNA migration mechanisms in cross-linked [7-9] and diluted gels [10-12] as well as artificial gels composed of microarrays [13-15]. Microfabrication techniques were also involved in the development of completely new concepts for DNA migration and separation, e.g., entropic traps [16-19] or molecular ratchets [20,21].

Our work focuses on the free solution separation of DNA in microfluidic devices using the influence of topographical structuring. The selection of microfluidic systems provides a huge pool of topographical variation, which can be achieved by conventional microstructuring techniques. Microchannels were fabricated by moulding of polydimethylsiloxane (PDMS) on a master wafer providing the inverted relief of the desired microstructures that were previously fabricated with an epoxy-based photoresist (SU-8) by contact lithography. Feature sizes in the range of the radius of gyration of the DNA molecules are produced. The mobility of $\lambda$-DNA (48 kbp) and T2-DNA (164 kbp) was investigated by real-time video recording of the migration of individual DNA molecules stained with the fluorescent intercalator YOYO.

\section{Materials and methods}

\subsection{Reagents}

PDMS (Sylgard 184) was purchased from Dow Corning (USA). SU-8 50 negative photoresist, thinner $\gamma$-butyrol acetone (GBL) and developer (propyleneglycolmethylether acetate) were obtained from Microresist (Germany). Si-wafers (P-Type 100, doped with boron) were from CrysTec (Germany). T2- and $\lambda$-DNA (164 and $48.5 \mathrm{kbp}$ ) were obtained from Fluka (Germany) and YOYO from Molecular Probes (USA). Tris buffer, $\beta$-mercapthoethanol and $\mathrm{NaCl}$ were obtained from Fluka and ethylenediaminetetraacetic acid (EDTA) from Sigma (Germany). $\mathrm{H}_{2} \mathrm{O}_{2}$ (30\%) and $\mathrm{H}_{2} \mathrm{SO}_{4}$ were from Merck (Germany). Water was deionised with a Milli-Q biocel (Millipore, USA). Cover glass slides $\left(76 \times 26 \mathrm{~mm}^{2}\right)$ were from Menzel Gläser (Germany).

\subsection{DNA staining}

DNA stock solutions were diluted in Tris buffer $(10 \mathrm{mM}$ Tris, $50 \mathrm{mM} \mathrm{NaCl}, 1 \mathrm{mM}$ EDTA, 2\% (v/v) $\beta$-mercapthoethanol, $\mathrm{pH} 8.3$ ) to a final concentration of $6 \mathrm{pM}$ dsDNA. YOYO was added to obtain a final dye to basepair ratio of about 1:7.5.

\subsection{Fabrication of master using photoplastic $S U-8$}

One hundred $\mathrm{ml}$ of SU-8 50 were diluted with $32.8 \mathrm{ml} \mathrm{GBL}$ to yield SU-8 5. After $24 \mathrm{~h}$ remaining bubbles could be removed by heating to $60{ }^{\circ} \mathrm{C}$ for $30 \mathrm{~min}$ on a hot plate. Si-wafers were cleaned with a standard piranha $\left(\mathrm{H}_{2} \mathrm{O}_{2}: \mathrm{H}_{2} \mathrm{SO}_{4}, 1: 3\right)$ etching procedure, dried on a spin coater and dehumided on a hot plate at $200{ }^{\circ} \mathrm{C}$ (HTZ-303D, ATV-Technologie, Germany) for $20 \mathrm{~min}$. The spin-coating process on 
a 1001/ST147 (Convac, Switzerland) consisted of (i) spreading the photoresist over the wafer at 700 $\mathrm{rpm}$ for $5 \mathrm{~s}$ and (ii) the spin-coat process at $5200 \mathrm{rpm}$ for $35 \mathrm{~s}$. The prebake process was performed by heating for $3 \mathrm{~min}$ at $40{ }^{\circ} \mathrm{C}, 3 \mathrm{~min}$ at $65^{\circ} \mathrm{C}$ and $5 \mathrm{~min}$ at $95{ }^{\circ} \mathrm{C}$. The i-line at $365 \mathrm{~nm}$ of a flood exposure unit (Modell 8011, Oriel, USA) was used to expose the photoresist through a chrome mask (DeltaMask, The Netherlands) in contact mode. The exposure dose for a 3- $\mu \mathrm{m}$ thick layer is roughly $110 \mathrm{~mJ} / \mathrm{cm}^{2}$. With a flux density of about $50 \mathrm{~mW} / \mathrm{cm}^{2}$ the optimised exposure time of $3.75 \mathrm{~s}$ was used.

For the post exposure bake, the temperature was first ramped to $40{ }^{\circ} \mathrm{C}$, then to $60{ }^{\circ} \mathrm{C}$, and finally $95^{\circ} \mathrm{C}$ and held for 1,3 and $20 \mathrm{~min}$, respectively. The coated wafer was then developed for $60 \mathrm{~s}$ in the developer bath. Rinses with acetone and isopropanole were performed before drying in a weak stream of nitrogen. A hard bake at $200{ }^{\circ} \mathrm{C}$ for 15 min followed and cooling down was performed on the hot plate.

\subsection{PDMS moulding}

Prepolymer and curing agent were mixed thoroughly in a ratio of 10:1, poured on the master wafer (see Fig. 1A) and fully polymerised within $3 \mathrm{~h}$ at $75^{\circ} \mathrm{C}$. The PDMS slab was then peeled off the master wafer and reservoir holes were punched from the structured side. A clean cover glass and a PDMS microchannel were oxidised for $5 \mathrm{~min}$ in an UVO cleaner (Model 42-220, Jelight, USA) and brought into contact immediately after oxidation. The channels were filled with $0.1 \mathrm{M} \mathrm{NaOH}$ which was replaced by Tris buffer solution after $1 \mathrm{~h}$.

\subsection{Channel characterisation}

A 20-nm thick gold layer was evaporated (MCS 10, Bal-Tec, FL) on the PDMS cast. Scanning electron microscopy (SEM) images of the structured microchannels were taken with a LEO 1538

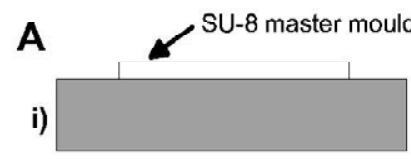

iii)

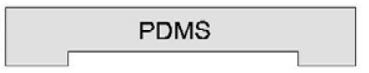

ii)

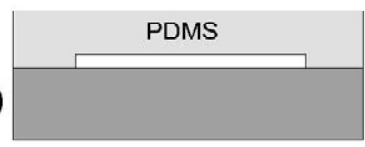

iv)

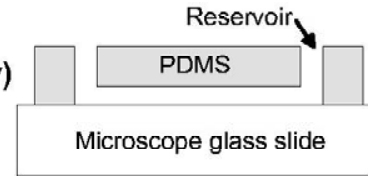

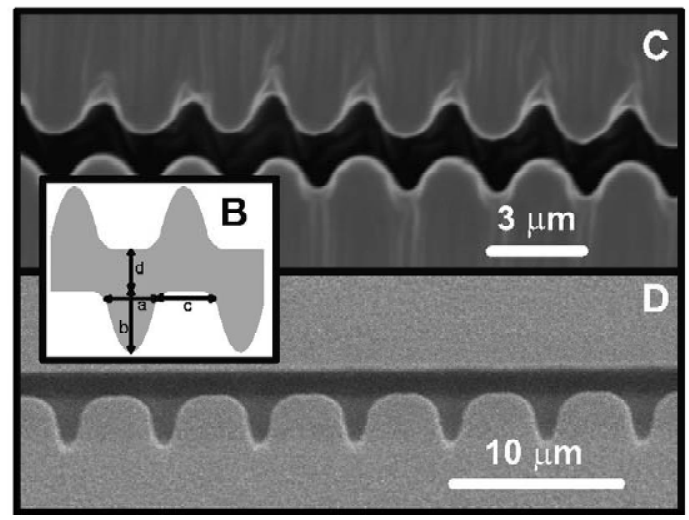

Fig. 1. (A) Process diagram of microchannel fabrication. (i) Convert shaped microchannel mould is fabricated with SU-8. (ii) PDMS is poured and cured over the mould. (iii) PDMS is released from the mould. The microchannel is thus replicated into PDMS. (iv) Reservoirs are punched through PDMS. The microchannel is closed with microscope glass slide. (B) The drawing describes the variables of the periodical cavities $(\mathrm{a}-\mathrm{d})$ in the microchannels. The channel width $d$ is used to define the channel layouts. (C) SEM top view of moulded $1.5 \mu \mathrm{m}$ and (D) $3 \mu \mathrm{m}$ PDMS microchannel. 
(LEO Electron microscopy, UK) at $5 \mathrm{kV}$ acceleration voltage and a JSM-880 (Jeol, JP) at $4 \mathrm{kV}$. The height of the structured master wafer was measured via profilometry (Dektak 3030ST, Veeco, USA).

\subsection{Fluorescence video microscopy}

The fluorescence video microscopy set-up consisted of an inverted microscope (Axiovert 100, Zeiss, Germany) with a filterset for fluorescence observation (XF100-3, Omega, USA), a $\times 100$ oil immersion objective (Plan Neofluar NA 1.3, Zeiss), a scientific interline transfer CCD-camera (Imager 3LS, LaVision, Germany) and a camera software for data analysis (DaVis 5.4.4, LaVision). A mercury arc lamp (HBO50, Zeiss) was used for excitation. The microfluidic device was mounted on a $x / y$-stage (99S008, Ludl Electronic Products, USA). A power supply (MCN 14-2000, FUG, Germany) connected to two platinum electrodes was used to apply the voltage.

\subsection{Data analysis}

The determination of the velocity of DNA molecules was carried out via cross-correlation analysis. With the help of command language macros (DaVis) the analysis was automated. Cross-correlating two subsequent images results in an intensity image, where the positions of the highest peaks represent the most probable displacements of the molecules. Several molecules with similar displacements superpose to one peak and therefore yield an averaged velocity. The histogram containing the averaged velocities of 199 data values (corresponding to 200 image correlations) was Gauss fitted.

\section{Results and discussion}

PDMS microstructures were fabricated by moulding on a SU-8 master wafer as shown in Fig. 1A and explained in detail in the experimental section. The master wafer was fabricated in a way that one or two side walls contained periodical cavities (see also Fig. 1B for details). Two different microchannel layouts have been used in this work: a 1.5- $\mu \mathrm{m}$ channel with periodical cavities on both lateral walls of the PDMS microchannel and a 3- $\mu \mathrm{m}$ channel with only one wall structured. Fig. 1C and D shows SEM images of these moulded PDMS microchannels.

The chrome mask used for the SU-8 master fabrication consisted of periodical square waveform structuring. For all moulded PDMS structures, the final periodical structures were slightly smaller than defined by the chrome mask dimensions and the periodical cavities were not a square waveform. This effect is attributed to the quality of the chrome mask, to the incomplete attachment of the mask to the SU-8 during exposure as a consequence of our experimental setup and to the diffraction limit. For the 1.5- $\mu \mathrm{m}$ channels the longitudinal periodicity $(a+c)$ was determined to $2.9 \mu \mathrm{m}$ and the lengths $b$ and $d$ were 1.0 and $0.8 \mu \mathrm{m}$, respectively. For the 3- $\mu \mathrm{m}$ structure the lengths $a$ and $c$ resulted in 3.0 and $2.8 \mu \mathrm{m}$, respectively, whereas $b$ and $d$ in 2.7 and $2.6 \mu \mathrm{m}$. The height of the master wafer structures was $2.7 \pm 0.1 \mu \mathrm{m}$ as confirmed by profilometry measurements.

The migration of $\lambda$-DNA (48 kbp) and T2-DNA (164 kbp) was investigated in structured and planar channels. DNA molecules were stained with YOYO in a basepair to YOYO ratio of 7.5:1 in Tris buffer, containing EDTA and mercaptoethanol. Single DNA molecules could be observed by 
fluorescence microscopy for at least $30 \mathrm{~s}$ without any fragmentation or significant loss in fluorescence intensity under these conditions. Only for low field strength, in which the migration velocity was a few micrometers per second, the molecules were several seconds under illumination in the typically $80-\mu \mathrm{m}$ wide observation window. Thus the fluorescence intensity and lifetime of the stained DNA molecule was always sufficient for the video microscopy experiments.

Fig. 2 demonstrates the migration of a single $\lambda$-DNA molecule in a $1.5-\mu \mathrm{m}$ channel at a field strength of $3 \mathrm{~V} / \mathrm{mm}$. The complete transit of the molecule through the $30.15-\mu \mathrm{m}$ display window is shown (450 pixels, 6.7- $\mu \mathrm{m}$ pixel size, $\times 100$ magnification). For the visualisation of the constrictions the channel was illuminated with a white light source with the accepted disadvantage of a higher background. However, the YOYO stained DNA molecules were still detectable with sufficient brightness and $S / N$-ratio.

Obviously the $\lambda$-DNA molecule is in a coiled conformation filling the periodical cavities of the microchannel. The diameter of a $\lambda$-DNA molecule in free solution can be estimated from the radius of gyration $R_{\mathrm{g}}=\left(1 / 3 l_{\mathrm{p}} L\right)^{1 / 2}$ according to the worm-like chain model [22], where $l_{\mathrm{p}}$ and $L$ denote persistence and contour length of the molecule, respectively. For YOYO stained $\lambda$-DNA Quake et al. [23] reported an increase in persistence length from 50 to $66 \mathrm{~nm}$ and an increase in contour length from 16 to $22 \mu \mathrm{m}$. Thus, for YOYO stained DNA the conformational diameter of the coiled $\lambda$-DNA molecules was estimated to $\sim 1.4 \mu \mathrm{m}$, corresponding to twice the calculated $R_{\mathrm{g}}$. Since lateral channel constrictions and diameter of the molecule are in the same dimension an influence on the conformation and the migration of the molecules can be expected, which is confirmed by the image sequence of Fig. 3. During its migration, the molecule was also found to be stretched spanning one period of cavities (see Fig. 2, pictures 11-13), but at any time its conformation was influenced by the channel constrictions. For the longer T2-DNA molecule a statistical molecular diameter of $2.6 \mu \mathrm{m}$ is calculated with a contour length of $72 \mu \mathrm{m}$ [24]. The influence of the constrictions on the conformation of the T2-DNA molecule can thus be expected to be stronger.

The mobilities (defined as the averaged velocity per electric field) of $\lambda$ - and T2-DNA were investigated consecutively in one microchannel. In Fig. 3 the mobility of the two molecules in a $1.5-\mu \mathrm{m}$ channel at increasing electric field is shown. The mobility for $\lambda$-DNA is always found

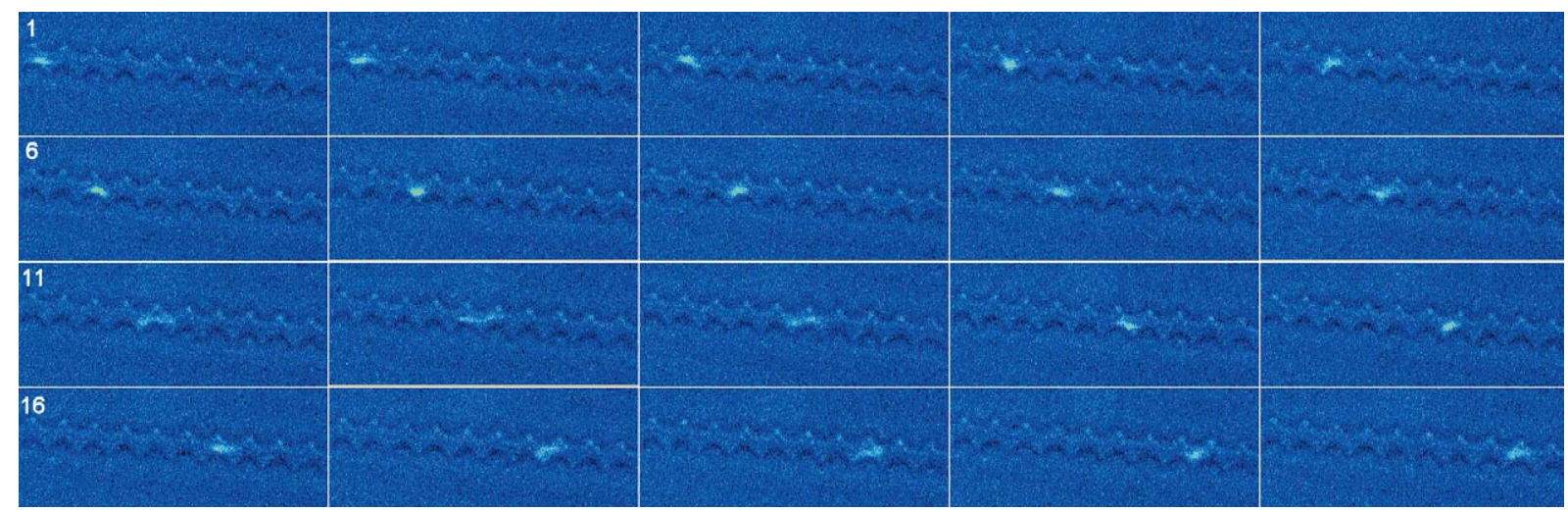

Fig. 2. Image sequence of a $\lambda$-DNA molecule migrating in a $1.5-\mu \mathrm{m}$ channel. The observation window corresponds to a microchannel section of $30.15 \mu \mathrm{m}$. The figure represents the real sequence with $40-\mathrm{ms}$ exposure and readout time between each image. The molecule requires $0.8 \mathrm{~s}$ to transverse the total observation window. 

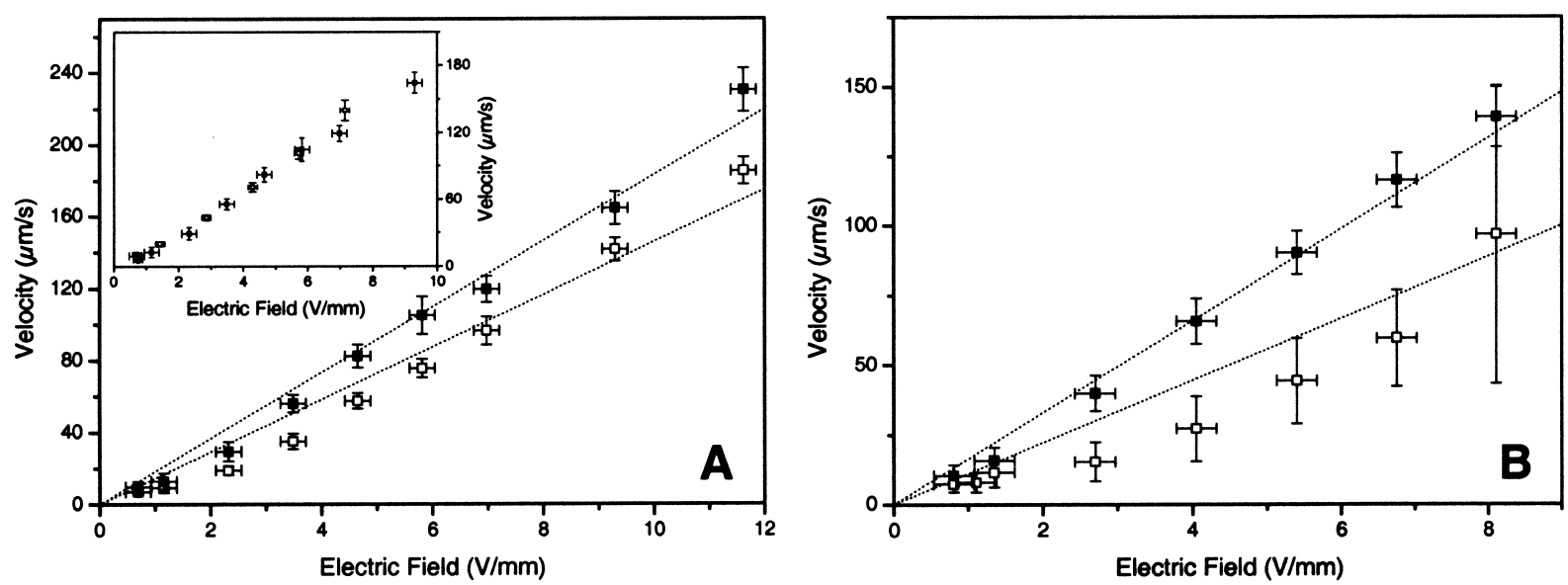

Fig. 3. Migration velocity of ( $\square$ ) $\lambda$-DNA and ( $\square$ ) T2 DNA (A) in a $1.5-\mu m$ and (B) in a $3-\mu m$ channel in dependence on the applied electric field. The $\lambda$-DNA molecules migrate significantly faster than T2-DNA for field strengths higher than 2 $\mathrm{V} / \mathrm{mm}$. The dotted lines represent a least-square linear fit of the data points. The inset of (A) shows the migration velocity of these two DNA types in a microchannel without periodical structuring $\left(20 \times 20-\mu \mathrm{m}^{2}\right.$ cross-section). The size dependence for the two molecules is lost.

significantly higher than for T2-DNA. For comparison, the free solution mobility in a planar microchannel without constrictions and with $20 \times 20 \mu \mathrm{m}^{2}$ cross-section was also investigated. Here, no difference in the migration velocity for the two DNA molecules could be observed (see inset of Fig. 3A) and a free solution mobility of $(1.84 \pm 0.03) \times 10^{-4} \mathrm{~cm}^{2} /$ Vs was determined.

For the $3-\mu \mathrm{m}$ channel, the channel constrictions in all three dimensions are in the range of the diameter of the T2-DNA molecule. The migration velocity as a function of the electric field in a $3-\mu \mathrm{m}$ channel is shown in Fig. 3B. As in the smaller channel configuration the $\lambda$-DNA migrates significantly faster than the longer T2-DNA.

It should be pointed out that the absolute mobility of DNA molecules could only be determined in the $20-\mu \mathrm{m}$ structures. In the topographically structured channels the mobility decreases steadily from experiment to experiment in the same micro channel. We attribute this effect to adsorption of DNA on the channel walls which could be observed by fluorescence microscopy and which is pronounced for higher surface to volume ratios. DNA adsorption increases the electroosmotic flow inverse to the migration direction and thus reduces the total migration velocity. To overcome this effect and to prove the length dependent mobility differences the order of the measurements was changed ( $\lambda$-DNA was measured before T2-DNA and vice versa). This always resulted in higher mobilities for $\lambda$-DNA over T2-DNA for one alternating measurement.

It is interesting to note that the migration velocity of the smaller $\lambda$-DNA in the two channel geometries is always faster than the larger T2-DNA molecules. This is in contrast to the case of entropic traps where the smaller molecules migrate slower than the larger [18,19]. For entropic trapping it is inevitable that the constrictions are in the dimension of the persistence length of the DNA molecules $(<100 \mathrm{~nm})$. The constrictions in our channels have the dimension of the diameter of the coiled DNA molecules $(1-3 \mu \mathrm{m})$ and thus do not exactly correspond to entropic traps. However, our experiments clearly demonstrated that the geometrically structured microchannels exhibit an influence on the migration velocity dependent on molecule length. The molecules are forced into 
conformations by the channel constrictions which influence their migration behaviour. Other effects arise from the adsorptive interaction of the molecules with the channel walls, which could be observed by fluorescence microscopy. Inhomogeneous electric field distributions in the microchannels as indicated by preliminary finite element simulations (data not shown) can also play a significant role in the migration mechanism. The combination of these effects is believed to cause a faster migration for shorter molecules.

\section{Conclusion}

Our work clearly demonstrates an alternative way to achieve length-dependent DNA migration and separation in gel free systems. PDMS microstructures with topographical structuring affects the migration of $\lambda$ - and T2-DNA molecules due to conformational effects. Individual DNA molecules could be observed and their pathways through the microstructures could be followed by real-time fluorescence video microscopy. Investigating migration velocities in structured channels showed that shorter $\lambda$-DNA migrated always significantly faster than T2-DNA. The comparison with planar channels clearly demonstrated, that without constrictions a length independent mobility is detected. The retardation of the longer T2-DNA molecules in the structured channels is attributed to entropic effects caused by conformational restrictions of the molecules, to surface interactions in the microchannels and to the distribution of the electric field in the microchannels. These phenomena will be the main issue for our future investigation on single molecule migration mechanisms. In order to investigate a larger variety of DNA sizes, an optimised microchannel geometry and a complete integrated microfluidic system with injection, separation and detection is under construction.

\section{Acknowledgements}

Financial support from the collaborative project SFB 613 from the Deutsche Forschungsgesellschaft (Teilprojekt D2) and technical support from Professor Heinzmann and PD Dr. Kleineberg (profilometry) and Dirk Meyners and Professor Reiss (SEM images) are gratefully acknowledged.

\section{References}

[1] S. Liu, Y. Shi, W.W. Ja, R.A. Mathies, Anal. Chem. 71 (1999) 566.

[2] A.T. Woolley, R.A. Mathies, Anal. Chem. 67 (1995) 3676.

[3] D. Schmalzing, N. Tsao, L. Koutny, D. Chisholm, A. Srinivasan, A. Adourian, L. Linton, P. McEwan, P. Matsudaira, D. Ehrlich, Genome Res. 9 (1999) 853.

[4] L. Koutny, D. Schmalzing, O. Salas-Solano, S. El-Difrawy, A. Adourian, S. Buonocore, K. Abbey, P. McEwan, P. Matsudaira, D. Ehrlich, Anal. Chem. 72 (2000) 3388.

[5] B.M. Paegel, C.A. Emrich, G.J. Wedemayer, J.R. Scherer, R.A. Mathies, Proc. Natl. Acad. Sci. USA 99 (2002) 574.

[6] J.-L. Viovy, Rev. Mod. Phys. 72 (2000) 813.

[7] D.C. Schwartz, M. Koval, Nature 338 (1989) 520.

[8] S. Gurrieri, S.B. Smith, K.S. Wells, I.D. Johnson, C. Bustamante, Nucleic Acids Res. 24 (1996) 4759.

[9] S.B. Smith, P.K. Aldridge, J.B. Callis, Science 243 (1989) 203. 
[10] X. Shi, R.W. Hammond, M.D. Morris, Anal. Chem. 67 (1995) 3219.

[11] X. Shi, R.W. Hammond, M.D. Morris, Anal. Chem. 67 (1995) 1132.

[12] J.J. Schwinefus, M.D. Morris, Macromolecules 32 (1999) 3678.

[13] W.D. Volkmuth, R.H. Austin, Nature 358 (1992) 600.

[14] O. Bakajin, T.A.J. Duke, J. Tegenfeldt, C.F. Chou, S.S. Chan, R.H. Austin, E.C. Cox, Anal. Chem. 73 (2002) 6053.

[15] S.W. Turner, A.M. Perez, A. Lopez, H.G. Craighead, Vac. Sci. Tech. B 16 (2002) 3835.

[16] J. Han, H.G. Craighead, Vac. Sci. Tech. A 17 (1999) 2142.

[17] J. Han, S.W. Turner, H.G. Craighead, Phys. Rev. Lett. 83 (2002) 1688.

[18] J. Han, H.G. Craighead, Science 288 (2000) 1026.

[19] J. Han, H.G. Craighead, Anal. Chem. 74 (2002) 394.

[20] J.S. Bader, R.W. Hammond, S.A. Henck, M.W. Deem, G.A. McDermott, J.M. Bustillo, J.W. Simpson, G.T. Mulhern, J.M. Rothberg, Proc. Natl. Acad. Sci. USA 96 (1999) 13165.

[21] R.W. Hammond, J.S. Bader, S.A. Henck, M.W. Deem, G.A. McDermott, J.M. Bustillo, J.M. Rothberg, Electrophoresis 21 (2000) 74.

[22] M. Doi, S.F. Edwards, in: Oxford Science Publications, Oxford, 1986.

[23] S.R. Quake, H. Babcock, S. Chu, Nature 388 (1997) 151.

[24] C. Carlsson, M. Jonsson, Macromolecules 29 (1996) 7802. 